\title{
Introduction to the special issue on Wigner functions
}

\author{
Mihail Nedjalkov $^{1}$ - Josef Weinbub ${ }^{1}$ - David K. Ferry ${ }^{2}$
}

Published online: 30 August 2015

(C) Springer Science+Business Media New York 2015

The Wigner function was first derived by E.P. Wigner around 1931 as an exotic outcome of the wave mechanics which, being defined in the phase space, does not favor either coordinate or momentum variables. Pioneered by H.J. Groenewold and J.E. Moyal the Wigner formulation of quantum mechanics evolved until the end of last century, to a fully autonomous, independent alternative to Hilbert space mechanics and path integral formulations. As S. MacLane put it (Ladies and) gentlemen: There is lots of room left in Hilbert Space, the same holds true for the phase space, where Wigner and alternative approaches, like Husimi and Bohmian distributions, are easily accommodated.

Applied to challenges in computational electronics, the Wigner approach offers the unique ability to describe transient problems for open systems by simultaneously accounting for different levels of approximation for the effects of the degrees of freedom associated with the environment. For example, the Wigner-Boltzmann equation enables the formalism with the efficient Boltzmann model of collisions (local in position/time) with phonons and impurities featuring the classical era of microelectronics. The equation bridges the gap between classical and quantum electron dynamics, ensuring a seamless transition between coherent and scattering dominated modes of transport. This approach

Mihail Nedjalkov

nedjalkov@iue.tuwien.ac.at

Josef Weinbub

weinbub@iue.tuwien.ac.at

David K. Ferry

ferry@asu.edu

1 Institute for Microelectronics, TU Wien, Gußhausstraße 27-29/E360, 1040 Wien, Austria

2 School of Electrical, Computer, and Energy Engineering, Arizona State University, Tempe, AZ 85287-5706, USA has been applied to simulate stationary and transient behavior of nanostructures, superlattices, to investigate processes of decoherence, quantum chaos, and recently beyond transport tasks in density functional theory and many body effects. The continuous accumulation of knowledge results in increased publication activities. A good example is the fact that two books by C. Jacoboni as well as D. Querlioz and P. Dollfus have been published in the year 2010, summarizing the theoretical and applied achievements of the Wigner research. Nevertheless, critics claim that, although various groups pick up the work, they abandon it again, never delivering a compelling research result. This criticism has been addressed by the Wigner community with the founding of the Wigner Initiative $^{1}$ in 2015 , bringing all research in this area closer together, thereby increasing synergy effects and fostering knowledge transfer.

This special issue is the first effort of the Initiative in this direction and comprises theoretical analysis, numerical aspects, and recent applications of the Wigner formalism, as well as comparisons with alternative phase space quasi-distribution functions and other quantum-mechanical approaches. In contrast to books, the purpose of which is to provide a systematic description, the contributions in this special issue rather highlight selected achievements in the field along with important logical and philosophical points in the phase space formulations of quantum mechanics. In particular, this special issue contains nine contributions characterized in the following:

I. Dimov et al. investigate the role of boundary conditions for the existence and the uniqueness of the solution of the stationary and transient formulations of the Wigner equation.

\footnotetext{
${ }^{1}$ http://www.iue.tuwien.ac.at/wigner-wiki/.
} 
D.K. Ferry provides an overview of different phase-space approaches with a special focus on quantum properties, such as entanglement, complemented by identifying core strengths of the Wigner approach.

B.J. Hiley discusses the close relationship between the Wigner-Moyal algebra and the original noncommutative quantum algebra introduced by von Neumann.

O. Jonasson et al. employ the Wigner function formalism to simulate partially coherent, dissipative electron transport in biased semiconductor superlattices, underlining the practical capabilities of Wigner function approaches.

M. Nedjalkov et al. introduce an analysis of formulations of the Wigner equation under a general gauge for the electric field, striving to develop alternative computational methods applied in the Wigner formalism.

E. Colomés et al. compare the Wigner, Husimi, and Bohmian distributions with respect to constructing a welldefined phase space distribution, providing a critical view on the properties of the individual formulations.
J.M. Sellier et al. analyzed a harmonic oscillator from the Wigner perspective. A brief review on utilizing a phase-space approach is given as well as the feasibility of the so-called signed particle Monte Carlo method is shown.

B.J. Spisak et al. apply the Wigner function to the description of the dynamics of conduction electrons in finite one-dimensional systems with randomly distributed scattering centers.

J. Weinbub et al. investigate parallelization strategies for two-dimensional Wigner Monte Carlo method based on the signed particle approach, enabling to utilize large-scale computing resources to stem the significant memory and computing demands.

As the guest editors of this special issue we would like to thank all authors for their contributions. 